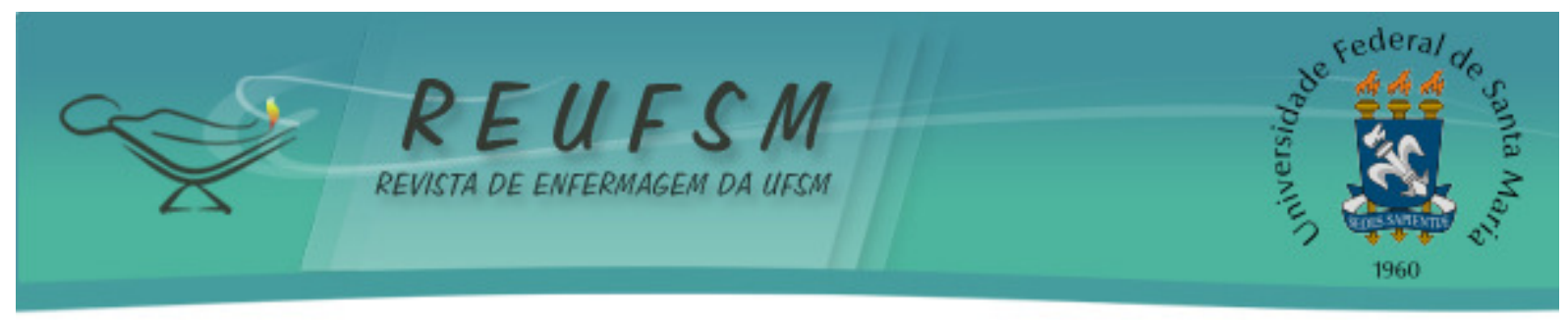

ARTIGO ORIGINAL

\title{
DENGUE: APLICAÇÃO DO PROTOCOLO DE ATENDIMENTO PELOS ENFERMEIROS ${ }^{1}$ \\ DENGUE: IMPLEMENTATION OF THE CARE PROTOCOL FOR NURSES \\ DENGUE: APLICACIÓN DEL PROTOCOLO DE ATENDIMIENTO POR LOS ENFERMEROS
}

\author{
Maria José Estanislau Daher ${ }^{2}$ \\ Bárbara Trindade do Bomfim Barreto ${ }^{3}$ \\ Silvia Cristina de Carvalho ${ }^{4}$
}

Doi: $10.5902 / 217976929069$

RESUMO: Objetivos: identificar o conhecimento dos enfermeiros de uma unidade de clínica da família sobre o protocolo de atendimento para dengue e descrever as dificuldades encontradas pelos enfermeiros em aplicar o protocolo da dengue. Método: pesquisa de campo do tipo descritiva com abordagem qualitativa. Coleta de dados realizada em uma Unidade de Clínica da Família, situada no bairro Jardim Novo, no Estado do Rio de Janeiro. Os sujeitos da pesquisa foram seis enfermeiros que compõem o quadro de funcionários da referida Unidade. Resultados: os dados foram agrupados e analisados em seu conteúdo, a fim de alcançar os objetivos propostos, destacando-se duas categorias de análise: pensamentos contraditórios e dificuldades expressas. Conclusão: confirmam existir o conhecimento dos protocolos, mas o atendimento, muitas vezes é automatizado, além do desconhecimento evidente da diferença entre os protocolos do Ministério da Saúde e do Rio de Janeiro.

Descritores: Dengue; Protocolos; Saúde da família.

ABSTRACT: Aim: to identify the knowledge of nurses in a clinical unit on family care protocol for dengue and to describe the difficulties encountered by nurses in applying the protocol dengue. Method: this is a field research with a descriptive qualitative approach. Data collection was performed on a unit Family Clinic, located in Jardim Novo, in the State of Rio de Janeiro. The subjects were 6 nurses who make up the staff of that unit. Results: after collection, the data were grouped and analyzed their content in order to achieve the proposed objectives, defining two categories of analysis: difficulties expressed and contradictory thoughts. Conclusion: confirm existing knowledge of protocols, but the service is often automated, besides the obvious lack of difference between the Ministry of Health and Rio de Janeiro.

Descriptors: Dengue; Protocols; Family health.

RESUMEN: Objetivos: identificar el conocimiento de los enfermeros de una unidad de Clínica de la Familia sobre el protocolo de atendimiento para el dengue y describir las dificultades encontradas por los enfermeros en aplicar el protocolo del dengue. Método: investigación descriptiva con el abordaje cualitativo. La recolección de datos se realizó en una Unidad de Clínica de la Familia, ubicada en el Estado de Rio de Janeiro. Los sujetos fueron 6 enfermeros que son funcionarios de esa unidad. Resultados: los datos fueron

\footnotetext{
${ }^{1}$ Artigo produzido a partir de Monografia de Conclusão de Curso de Graduação em Enfermagem.

${ }^{2}$ Coordenadora e docente da pós Graduação em Enfermagem do Trabalho da Universidade do Grande Rio UNIGRANRIO. Mestre em Enfermagem pela UniRio (2000). Universidade do Grande Rio - UNIGRANRIO, Duque de Caxias, RJ, Brasil. Email: maria_daher@ymail.com

${ }^{3}$ Enfermeira. Graduada pela Universidade do Grande Rio - UNIGRANRIO, Rio de Janeiro, RJ, Brasil. Email: barbara_trin@ig.com.br

${ }^{4}$ Professora adjunta da Universidade do Grande Rio - UNIGRANRIO. Mestre em Enfermagem pela Universidade do Estado do Rio de Janeiro (2000). Doutoranda do Instituto de Estudos em Saúde Coletiva da UFRJ. Universidade do Grande Rio - UNIGRANRIO, Duque de Caxias, RJ, Brasil. Email: silviac_carvalho@yahoo.com.br
} 


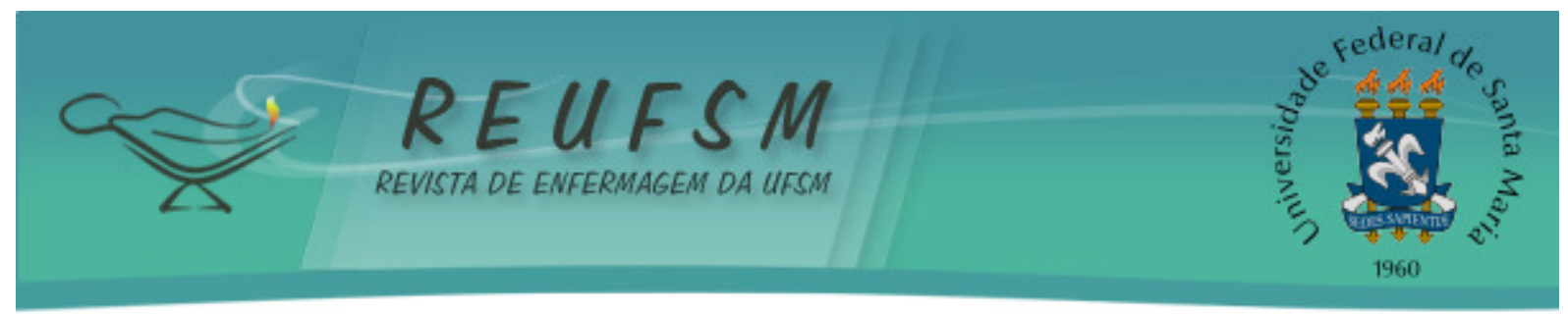

agrupados y analizados en su contenido con el fin de alcanzar los objetivos propuestos, destacando dos categorías de análisis: dificultades expresas y pensamientos contradictorios. Conclusión: confirman existir el conocimiento de los protocolos, pero el servicio es a menudo automatizado, además de la evidente falta de diferencia entre los protocolos y el Ministerio de la Salud y del Rio de Janeiro.

Descriptores: Dengue; Protocolos; Salud de la família.

\section{INTRODUÇÃO}

A dengue é um dos principais problemas de saúde pública no mundo. A Organização Mundial da Saúde (OMS) estima que 80 milhões de pessoas se infectem anualmente, em 100 países, de todos os continentes, exceto a Europa. ${ }^{1}$

Cerca de 550 mil doentes necessitam de hospitalização e 20 mil morrem em consequência da dengue. A disseminação do Aedes aegypti depende de condições favoráveis fruto da urbanização acelerada que criou cidades com deficiências de abastecimento de água e de limpeza urbana; pela intensa utilização de materiais não-biodegradáveis, como recipientes descartáveis de plástico e vidro; e pelas mudanças climáticas. ${ }^{2}$

A dengue foi vista pela primeira vez no mundo no final do século XVIII, no Sudoeste Asiático, em Java, e nos Estados Unidos, na Filadélfia. No entanto, só foi reconhecida como doença no século XX. 0 primeiro caso de febre hemorrágica da dengue surgiu na década de 50, nas Filipinas e Tailândia. Após a década de 60, a presença do vírus intensificou-se nas Américas. Pesquisadores identificaram vários sorotipos da doença, numerados de um a quatro. O segundo surto ocorreu na Venezuela, em 1989. No Brasil, há referências de epidemias desde 1916, em São Paulo, e em 1923, em Niterói, no Rio de Janeiro (RJ), sem comprovação laboratorial. Entre os anos de 1981 e 1982, ocorreu a primeira epidemia, em Boa Vista, Roraima, causada pelos sorotipos um e quatro, considerados mais perigosos. A partir de 1986, ocorreram epidemias, atingindo o Rio de Janeiro e algumas capitais da região Nordeste. ${ }^{3}$

Em nosso país, as condições socioambientais favoráveis à expansão do Aedes aegypti possibilitaram uma dispersão desse vetor, desde sua reintrodução em 1976, que não conseguiu ser controlada com os métodos tradicionalmente empregados no combate às doenças transmitidas por vetores, em nosso país e no continente. Programas essencialmente centrados no combate químico, com baixíssima ou mesmo nenhuma participação da comunidade, sem integração intersetorial e com pequena utilização do instrumental epidemiológico, mostraram-se incapazes de conter um vetor com altíssima capacidade de adaptação ao novo ambiente criado pela urbanização acelerada e pelos novos hábitos. ${ }^{2}$

A dengue provocou dois picos endêmicos no Município do Rio de janeiro (2002 e 2008). As áreas mais críticas apresentavam terrenos baldios, casas abandonadas, dentre outros fatores que contribuíram para a promoção de criadouros. As Áreas de Planejamento (AP) mais afetadas foram da AP1 a AP5, sendo o Centro, Tijuca, Bonsucesso, Taquara e Santa Cruz os Bairros mais atingidos com 1585, 4139, 2832, 4203 e 14391 casos, respectivamente. A faixa etária mais atingida foi de 15 a 49 anos, parcela ativa da população, que se movimenta mais dentro do Município e está mais exposta à doença. ${ }^{4}$

A organização do trabalho em saúde perpassa por momentos de crise como, por exemplo, as epidemias, fazendo-se necessário uma reavaliação do processo de trabalho. Nesse sentido, a classificação de risco do paciente com dengue surge com o intuito de diminuir o tempo de espera no serviço de saúde. Para essa classificação, foram utilizados os critérios da Política Nacional de Humanização do Ministério da Saúde e o estadiamento da doença. ${ }^{5}$

É importante que o enfermeiro realize todas as etapas da anamnese e do exame físico para que não ocorra equivoco na classificação da Dengue e, posteriormente, nos 


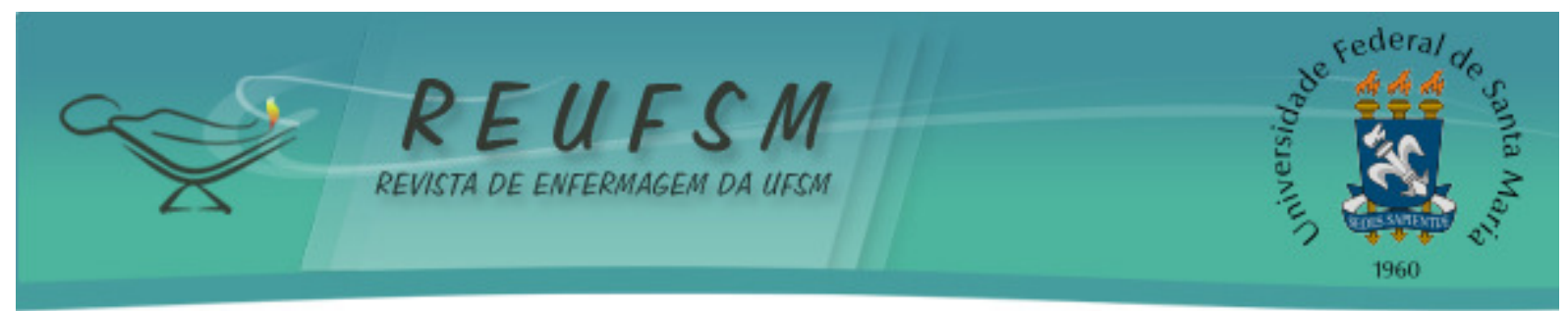

cuidados de enfermagem, que devem ser individualizados. Nesse caso, torna-se necessário que nenhum processo seja perdido ao longo do caminho como, por exemplo, realizar exame físico completo a cada retorno do cliente à unidade de saúde, a fim de observar a evolução e/ou regressão da doença e identificar caso ocorra algum sinal/sintoma. 0 registro de todas as atividades é uma prova contundente de que o protocolo foi seguido. ${ }^{6}$

Os protocolos de combate aos casos de Dengue organizados pelo Ministério da Saúde e Municípios sugerem passo a passo do que deve ser realizado em casos confirmados ou de suspeita da doença. No entanto, não se tem certeza da sua aplicabilidade, na prática, pelos enfermeiros. Capacitações, atualizações e estudos constantes são necessários para que os atendimentos não sejam negligenciados ou efetuados de forma incompleta.

Diante do exposto, essa pesquisa apresenta como objeto: o estudo da aplicabilidade, pelo enfermeiro, do protocolo da dengue. As questões que instigaram a construção do projeto foram: quais ações podem ser realizadas de forma a sensibilizar os enfermeiros sobre a magnitude da doença? Quais as dificuldades para executar o protocolo de dengue?

Objetiva-se identificar o conhecimento dos enfermeiros de uma unidade de clínica da família sobre o protocolo de atendimento para dengue e, especificamente, descrever as dificuldades encontradas pelos profissionais enfermeiros em aplicar o protocolo da dengue. Esse estudo justifica-se pelos constantes casos de dengue que ocorrem em nosso país e as dificuldades encontradas pela população e profissionais de saúde em seu combate. Torna-se relevante para a área de saúde, no que tange ao conhecimento, conscientização e combate da dengue.

\section{MÉTODO}

O presente estudo trata-se de uma pesquisa de campo do tipo qualitativa e descritiva. A análise qualitativa tem como objeto situações complexas ou particulares. Estes estudos podem descrever a complexidade de determinado problema, analisar a interação de certas variáveis, compreender e classificar processos dinâmicos vividos por grupos sociais, contribuir no processo de mudança de determinado grupo e possibilitar, em maior nível de profundidade, o entendimento das particularidades do comportamento dos indivíduos. ${ }^{7}$

A pesquisa descritiva objetiva a descrição das características de determinada população ou fenômeno, ou visa estabelecer relações entre variáveis, que podem ser obtidas através da utilização de técnicas de coleta de dados. ${ }^{8}$

A coleta de dados foi realizada em uma Unidade de Clínica da Família, situada no bairro Jardim Novo, no Estado do Rio de Janeiro. A Unidade foi escolhida porque dispõe de uma boa demanda de público alvo, ou seja, um alto número de casos de dengue, o que propicia a aplicação do protocolo de dengue pelo enfermeiro aos clientes cadastrados na clínica e que buscam atendimento. Os sujeitos do estudo foram os enfermeiros da Clínica da Família que compõem o quadro de profissionais da referida unidade. Para a coleta de dados, foi utilizado um instrumento de pesquisa elaborado pelos pesquisadores, que se constituiu em uma entrevista padronizada ou estruturada. A entrevista padronizada ou estruturada deve seguir um roteiro previamente estabelecido, ou seja, as perguntas feitas ao indivíduo são predeterminadas. ${ }^{9}$

A entrevista foi realizada na própria unidade, na qual um entrevistador aplicou um instrumento, mediante a assinatura prévia do termo de consentimento livre e esclarecido pelo entrevistado, em cumprimento a todas as exigências éticas e legais. A coleta foi iniciada no segundo semestre do ano de 2012, no mês de novembro, após autorização da Unidade Sediadora e aprovação do Comitê de Ética e Pesquisa (CEP) da Universidade do Grande Rio, sob o número 141.881. Os dados foram coletados até atingirem o quantitativo de seis enfermeiros, que compõem a equipe da Unidade. No instrumento de coleta de dados, foram atribuídos códigos aos profissionais, de modo a preservar o seu anonimato. 


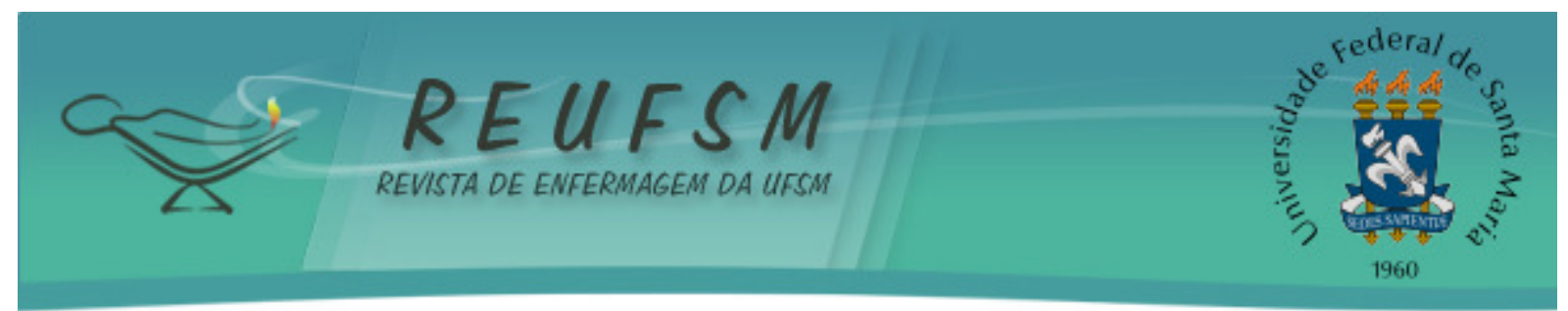

Foram entrevistados seis enfermeiros de uma Clínica da Família sobre o protocolo de atendimento à dengue. As apresentações das entrevistas seguem esta ordem: o primeiro enfermeiro que foi entrevistado foi definido com a sigla ENF1; o segundo ENF2; o terceiro, ENF3; o quarto, ENF4; o quinto, ENF5; e o sexto, ENF6; respectivamente. Com a finalização das entrevistas, o conteúdo adquirido foi transcrito e lido até que fosse possível o agrupamento e a comparação dos dados para categorização e análise, que teve o intuito de atender aos objetivos dessa pesquisa.

\section{RESULTADOS E DISCUSSÃO}

A análise de dados foi contundente e definiu duas categorias, mediante processo de leitura e releitura das entrevistas e associação com os objetivos propostos no estudo. São elas: pensamentos contraditórios e dificuldades expressas. As categorias agregam mensagens e são empregadas na análise de dados para uma organização que permite classificar, agrupar ideias, buscando expressões que se repetem e se relacionam. ${ }^{10-11}$

A proliferação da dengue está diretamente relacionada com as condições climáticas, logo se tornou um dos principais problemas de saúde pública no mundo. Existem doenças que são introduzidas pelo clima em tempos diferentes e afetam o homem. Dessa forma, demonstram correlações íntimas com as condições climáticas e com a estação do ano. O principal transmissor da dengue, o mosquito Aedes aegypti, é totalmente adaptado ao ambiente urbano e acha, junto aos domicílios humanos, as condições necessárias para o seu desenvolvimento, que ocorre em águas acumuladas em recipientes que, em sua maioria, são usados pelo homem. Além disso, as áreas que mantém temperaturas mais elevadas são mais suscetíveis à presença e proliferação do mosquito Aedes aegypti. Considera-se, então, a dengue uma doença tropical. ${ }^{12}$

A cidade do Rio de Janeiro, de clima tropical, possui uma população de 6,323 milhões de habitantes. É conhecida por seu crescimento urbano desordenado e favelização, reforçados pelo elevado grau de desigualdade social, no qual famílias vivem em condições precárias de vida, em lugares sem saneamento básico e com presença de focos de dengue. Esses fatos contribuem para a vulnerabilidade, para o aparecimento de novas doenças e a reintrodução de outras já erradicadas. ${ }^{12-13}$

\section{Pensamentos contraditórios}

Essa categoria destaca as respostas contraditórias dos entrevistados diante do que deveria ser uma atuação hábil que pode salvar vidas, ou interromper um processo de adoecimento. Analisando as questões em que se investiga sobre o conhecimento do profissional enfermeiro sobre o protocolo de atendimento à dengue e qual o protocolo utilizado em sua unidade de saúde, respectivamente, foi visto que os seis enfermeiros conhecem o protocolo de dengue, porém não demonstraram certeza nas respostas, quando perguntados sobre o protocolo utilizado em sua unidade de saúde. Além disso, todos desconhecem se existe ou não diferença entre o protocolo da dengue preconizado pelo Ministério da Saúde e o utilizado no Município do Rio de Janeiro.

Identificar precocemente os casos de dengue faz-se importante para a tomada de decisões e a implementação de medidas imediatas, objetivando o controle da doença. Nesse contexto, a conscientização e o conhecimento do protocolo tornam-se relevantes para o profissional enfermeiro, se o objetivo é o combate à doença. 0 desconhecimento do processo que envolve o tratamento da dengue e que foi, a princípio, determinado pelo Ministério da Saúde, pode conduzir o profissional a uma insegurança no atendimento 


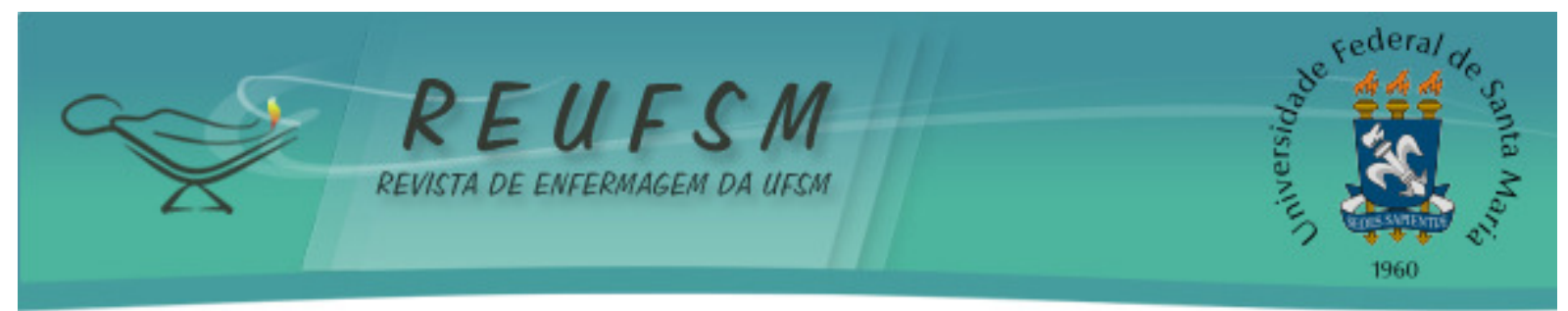

imediato, ou torná-lo mecanizado, remetendo o enfermeiro meramente a uma atuação técnica, dissociada de fundamentação teórica. de Saúde:

Destaca-se nas entrevistas dos ENF2 e ENF6, sobre o protocolo em uso na Unidade

[...] Existiu treinamento e capacitação sobre o protocolo sim. (ENF2)

\section{[...] Não me lembro de capacitação ou treinamento. (ENF6)}

Ambos profissionais foram admitidos na Unidade na mesma época e dizem aplicar integralmente o protocolo, apesar das respostas não coincidirem.

A Estratégia de Saúde da Família propõe mudanças de paradigma e pretende tornar o trabalho em saúde mais humanizado e holístico. ${ }^{12} \mathrm{Na} E S F$, o profissional estabelece vínculo com o usuário, o que facilita os atendimentos, seja em consultório ou em domicílio. A ausência desse vínculo interfere no atendimento e prejudica os resultados. A dengue pode apresentar uma evolução rápida, de um estágio a outro e o manejo adequado pode tornar-se vital. ${ }^{13}$ Essa dinamicidade na ESF permite uma aproximação mais efetiva do doente que, no entanto, pode esbarrar na falta de conteúdo do profissional enfermeiro.

O Protocolo de Enfermagem na Atenção Primária à Saúde é uma ação da Secretaria Municipal de Saúde e do Conselho Regional de Enfermagem (COREN)-RJ que uniformiza o atendimento da rede básica de saúde do Município do Rio de Janeiro. Ele atende as determinações da lei 7498/86 e garante o desempenho dos enfermeiros, visando às necessidades da população. ${ }^{14}$

0 atual protocolo para cuidados e tratamento para dengue do Município do Rio de Janeiro para enfermeiros é baseado e padronizado a partir do Manual da Dengue: Diagnóstico e manejo clínico adulto e criança do Ministério da Saúde, 2011. Logo, não existem diferenças profundas entre eles. No entanto, torna-se necessário realizar, investir e estimular a inserção da educação continuada para os enfermeiros da ESF e incentivar a educação permanente sobre, não somente o protocolo de dengue, mas também sobre todos os protocolos existentes pertinentes ao papel do enfermeiro. enfermeiros:

A incerteza relacionada ao uso do protocolo fica clara nas colocações dos

[...] Penso que minhas ações estão corretas, pois há muito atendo pacientes com dengue. Nós recebemos informes da secretaria com o que deve ser feito, não me preocupo em ler o protocolo. Não sei qual usam aqui, acho que do Ministério. (ENF 3)

[...] Na maior parte não tenho tempo de estudar, pois trabalho em outros lugares, acho que atualizações deveriam ser feitas, mas aqui na Unidade eu nunca fiz. (ENF 4)

Observou-se, na pesquisa, desconhecimento dos enfermeiros e ausência de buscas pessoais sobre os protocolos e suas diferenças. Os enfermeiros apesar de argumentarem diferenciadamente sobre o protocolo da dengue utilizado na Unidade de Saúde deixam claro que a base desse protocolo é do Ministério da Saúde. No entanto, a falta de conscientização e conhecimento, em algumas respostas, podem provocar dúvidas e, até mesmo, falhas de atendimento. A educação permanente em enfermagem faz parte de uma realidade que compromete a prática assistencial, quando não efetivada, e merece atenção por parte dos profissionais que pretendam exercer a sua profissão com qualidade, sendo pró-ativos e engajados na sociedade. 


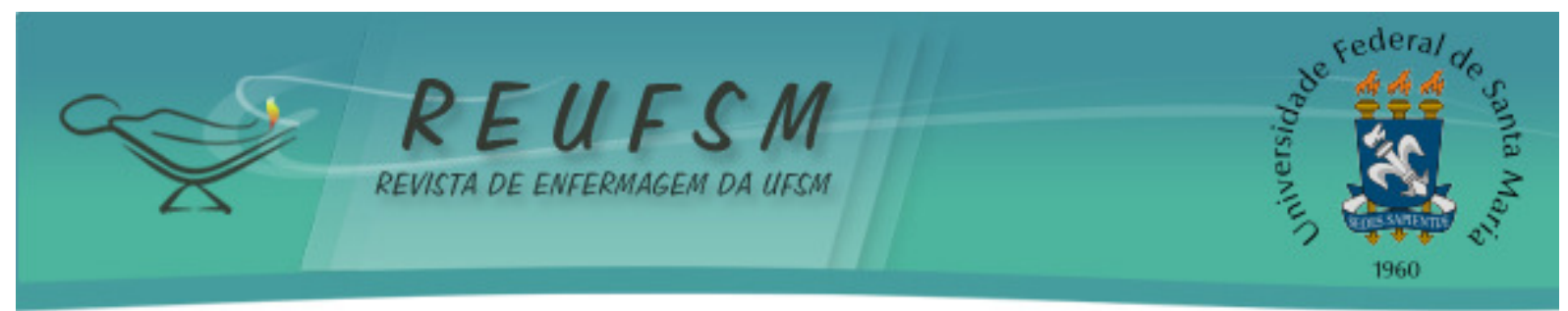

As contradições relacionadas ao conhecimento do protocolo da dengue por parte dos enfermeiros são reforçadas, também, na fragilidade do Sistema Único de Saúde (SUS), que gera insatisfação dos usuários com as altas demandas de atendimento, o número reduzido de enfermeiros e a demora para conseguir os agendamentos. Os relatos dos sujeitos da pesquisa reforçam a alta demanda de atendimento como um fator dificultador de capacitações:

[...] Não temos tempo para estudar, aqui tudo é muito rápido e em época de dengue, tudo fica mais complicado. Temos que nos virar. Não sei que protocolo usam, deve ser o do Rio, que é mais novo, né? (ENF 4)

[...] Como recebemos os informes da secretaria eu confio $e$ trabalho. O tempo é curto e tem muita gente para atender. Imagino que esses informes sejam baseados no protocolo do Ministério. Eu já atendo dengue tem tempo. (ENF 5)

Estruturar uma proposta de educação permanente institucional constitui-se em uma das alternativas de rever o processo de trabalho, que justifica-se por ser uma das formas de qualificação dos trabalhadores em saúde e oferta de uma assistência mais resolutiva. ${ }^{15} \mathrm{~A}$ dengue não é uma patologia desconhecida, no entanto o manejo adequado tem como principal objetivo evitar um possível agravamento do quadro clínico do paciente que procura os serviços de diagnóstico e tratamento e, consequentemente, prevenir óbitos pela doença.

\section{Dificuldades expressas}

O enfermeiro é habilitado e apto para a realização do acolhimento e classificação de risco do usuário, logo é fundamental saber aplicar a sistematização da assistência de enfermagem (SAE) em todas as suas etapas. No entanto, esse profissional, muitas vezes, vive dificuldades com relação ao seu campo de ação, o que aprende, enquanto acadêmico e a realidade de sua prática, gerando tensões em seu universo de atuação. ${ }^{16}$

Analisando a questão na qual investiga-se sobre as possíveis dificuldades encontradas pelo enfermeiro em aplicar o protocolo de atendimento a dengue, destacamse como principais dificuldades:

\section{[...] A classificação dos sinais e sintomas [...]. (ENF3; ENF4)}

[...] observo dificuldade em aplicar o protocolo de dengue, mas também em realizar a sistematização da assistência de enfermagem como um todo; há sobrecarga de trabalho; e há falta de disponibilidade de horário para realizar uma assistência integral ao cliente. (ENF1)

[...] Eu não penso, vou fazendo o atendimento, na hora não me vem à cabeça olhar o protocolo e ver se está certo. Minha dificuldade é essa. (ENF 2)

[...] Seguir o passo a passo do protocolo. Aqui é difícil pela alta demanda. (ENF 5)

[...] Acho que sigo o protocolo, mas é automático, não paro para ler, então às vezes tenho dúvidas. (ENF 6) 


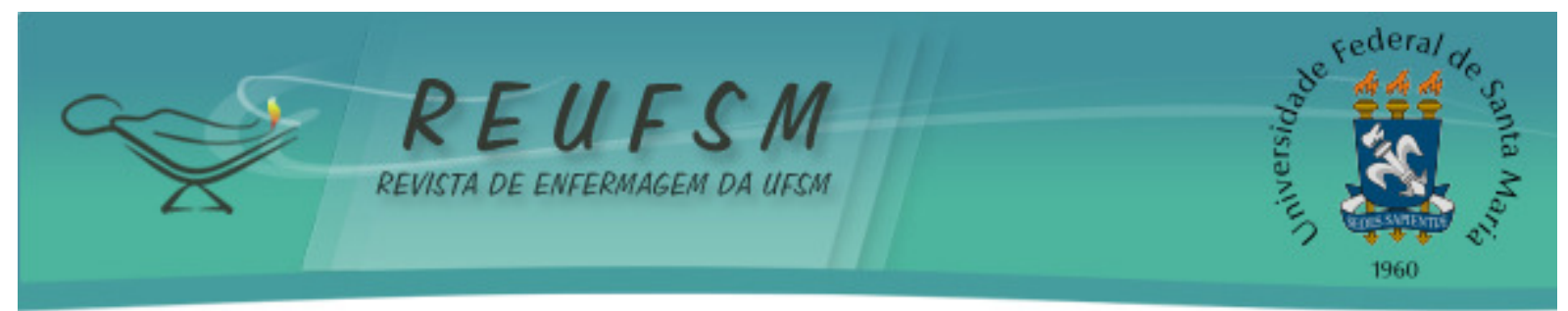

Dos enfermeiros entrevistados, três afirmam que não aplicam integralmente o protocolo, seguindo o atendimento a partir da sintomatologia do usuário e que isso é motivado pela falta de tempo e grande demanda. Isto implica no conhecimento prévio das intervenções imediatas e da doença.

Sabe-se que, em épocas epidêmicas os polos de atendimento da dengue permanecem cheios, não havendo número de profissionais suficientes para atender e realizar a consulta de enfermagem, adequadamente, devido à demanda de usuários que procuram os serviços de saúde com suspeita de doença. Muitas vezes, esses funcionários acumulam funções técnicas e administrativas. De acordo com o protocolo, o enfermeiro deve se equilibrar entre as funções clínicas e gerenciais. 0 aumento das funções gerenciais pode fragilizar a confiança dos usuários em sua capacidade técnica, pela sua ausência, o que dificulta a formação de vínculo. ${ }^{14}$

É importante destacar o relato do ENF 6, relacionado ao acúmulo de funções, que reforça a citação supracitada:

[...] Somos muito cobrados, pois temos que fazer os atendimentos em todas as áreas e ainda dar conta do gerenciamento. Em época de dengue fica caótico. Quando chega a época de fechar as estatísticas, isso piora e somos cobrados até pelos usuários. (ENF 6)

Propor uma agenda de pesquisa pode ser uma solução viável, quando a pretensão é manter contato com a qualidade e a eficácia do atendimento para a dengue. Dessa forma, o conhecimento do estadiamento clínico da doença, o estudo das formas mais graves e pesquisas para o desenvolvimento de vacinas são medidas que devem ser incentivadas. ${ }^{17}$

Apesar do avanço do SUS, no campo da vigilância e prevenção das doenças infecciosas e do empenho de muitos gestores deste sistema para controlar este problema, a introdução do sorotipo Dengue (DEN)-4, no Brasil, agravou o cenário epidemiológico da dengue, em 2010. A população estava completamente suscetível às infecções por este sorotipo e, além disso, a demanda aos serviços de saúde tornou-se tão elevada que ficou difícil prestar atendimento de qualidade a todos que necessitavam. É notável que as experiências anteriores, vide epidemia ocorrida em 2008, no Rio de Janeiro, apoiam essa previsão de caos. ${ }^{18}$

[...] O ideal seria contratação extra ou remanejamento de enfermeiros em épocas de dengue. Dessa forma, poderíamos ter um enfermeiro atendendo somente os casos de dengue, isso seria ideal. (ENF2)

A afirmação do ENF 2 demonstra a fragilidade que ainda existe nas Unidades de Clínica da Família ao deparar-se com uma epidemia de dengue. 0 relato de impotência implícito nas falas das enfermeiras, sujeitos do estudo, limita o atendimento e pode comprometer a qualidade das ações de enfermagem e a profissão. Essa impotência é vivenciada, também, pela população, que se sente limitada quanto aos aspectos biológicos, sociais e comportamentais da dengue, necessitando de suporte teórico constante dos profissionais de saúde. ${ }^{18}$

Justificar a falta de funcionários à grande demanda de usuários e o acúmulo de funções à não aplicação de um protocolo que pode salvar vidas, minoriza a profissão. Deve-se priorizar o atendimento de acordo com a avaliação da classificação de risco, seguindo uma normatização pré-aprovada e segura, incluindo a palavra qualidade no vocabulário da assistência de enfermagem. A conscientização do trabalho realizado e a responsabilidade que o enfermeiro detém, no momento do atendimento do doente, merecem atenção. Contudo, alguns fatores corroboram para o comportamento desses 


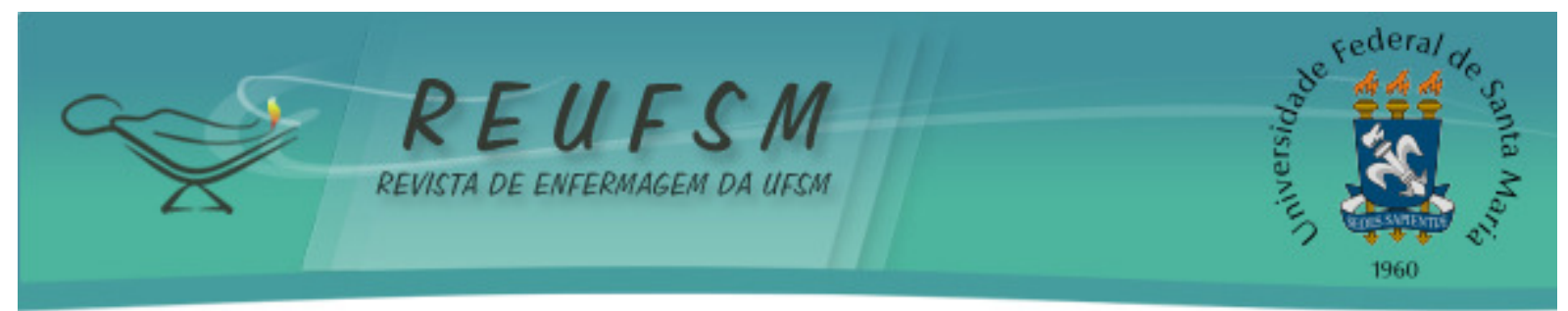

profissionais e devem ser pensados, destacando-se os baixos salários, que geram acúmulos de vínculos empregatícios e a carência de profissionais qualificados.

\section{CONCLUSÃO}

Esta pesquisa oportunizou discutir sobre a aplicabilidade do protocolo de atendimento à dengue pelo profissional enfermeiro. Partindo do pressuposto que a aplicação do protocolo de atendimento à dengue, durante a consulta de enfermagem em seu uso correto pode salvar vidas, procurou-se demonstrar se essa conduta realmente é seguida.

Foram analisados os dados obtidos junto aos enfermeiros e, a partir daí, identificouse o conhecimento dos mesmos sobre o protocolo e as dificuldades apresentadas pelos profissionais em estarem aplicando-o no dia-a-dia, durante o atendimento/consulta de enfermagem. Verificou-se que todos os enfermeiros entrevistados conhecem o protocolo de atendimento à dengue, porém alguns referem não ter tido treinamento/capacitação para utilização dos protocolos referidos nesse estudo. Foi percebido o desconhecimento dos enfermeiros sobre a existência de dois tipos de protocolos, sendo esses, o protocolo preconizado pelo Ministério da Saúde e, o outro, do Município do Rio de Janeiro. Logo, ocorreram diferenças entre os enfermeiros e o uso dos protocolos na unidade em que trabalham pela falta de conhecimento sobre a existência desses dois protocolos.

Esses profissionais apontaram dificuldades em aplicar o protocolo, sendo o foco do atendimento a classificação dos sinais e sintomas. Dessa forma, a sistematização da assistência de enfermagem, como um todo, a sobrecarga de trabalho e a falta de disponibilidade de horário para realizar uma assistência integral ao usuário, foram justificativas para a não utilização dos passos do protocolo. Ações de educação em saúde, nesse contexto, são também dificultadas pela falta de tempo, lembrando que a melhor forma de se evitar a dengue ainda é combater os focos de acúmulo de água, locais propícios para a criação do mosquito transmissor da doença. Para tanto, a prevenção torna-se essencial.

Nessa pesquisa, evidencia-se a importância em realizar, investir e estimular a inserção da educação continuada no cotidiano do trabalho do enfermeiro da Estratégia Saúde da Família e, igualmente, incentivar espaços de educação permanente em relação ao protocolo da dengue, bem como sobre todos os temas pertinentes ao papel do enfermeiro. O objetivo deve estar direcionado a melhora e/ou implantação da sistematização da assistência de enfermagem a cada usuário, a fim de reduzir os riscos de agravos da dengue e de outras doenças, realizar prognósticos e salvar vidas.

\section{REFERÊNCIAS}

1. Ministério da Saúde (BR). Portal da Saúde. Dengue [Internet]. [acesso em 2012 out 2]. Disponível em: http://portal.saude.gov.br/portal/svs/visualizar_texto.cfm?idtxt=22207.

2. Brasil. Ministério da Saúde/Fundação Nacional da Saúde. Vigilância Epidemiológica. Programa Nacional de Controle da Dengue (PNCD). Brasília (DF): Ministério da Saúde; 2002.

3. Ministério da Saúde (BR). Portal da Saúde. Vigilância Epidemiológica [Internet]. [acesso em 2012 out 3]. Disponível em: http://portal.saude.gov.br/portal/saude/visualizar_texto.cfm?idtxt=21389.

4. Daher MJE, Carvalho JA, Grasel R, Ferreira VN. A dengue no município do Rio de Janeiro: aspectos epidemiológicos dos últimos oito anos. Rev Rede de Cuidados em Saúde. 2010;4(2). 


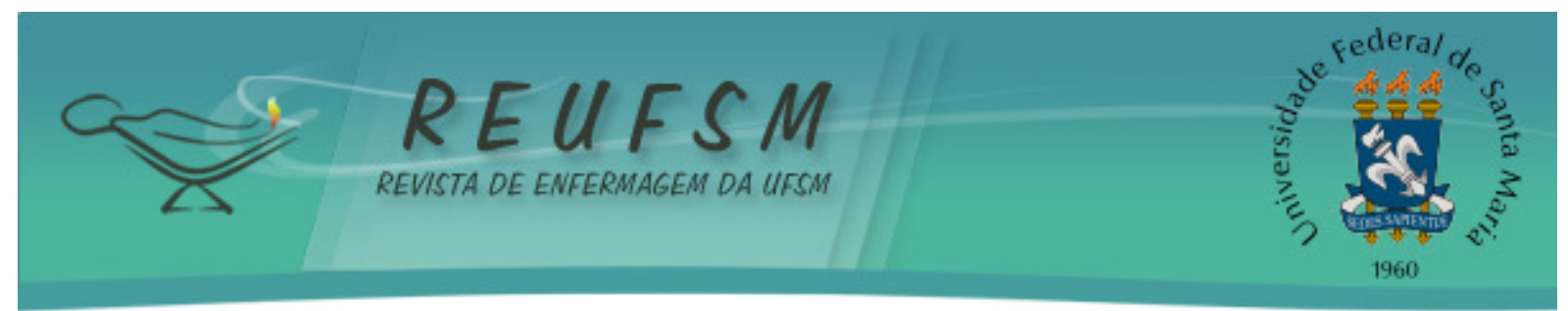

5. Brasil. Ministério da Saúde. Secretaria de Vigilância em Saúde. Diretoria Técnica de Gestão. Dengue: diagnóstico e manejo clínico: adulto e criança. $4^{a}$ ed. Brasília (DF): Ministério da Saúde; 2011. (Série A. Normas e Manuais Técnicos).

6. Brasil. Ministério da Saúde. Secretaria de Vigilância em Saúde. Diretoria Técnica de Gestão. Dengue: diagnóstico e manejo clínico: adulto e criança. $3^{a}$ ed. Brasília (DF): Ministério da Saúde; 2007. (Série A. Normas e Manuais Técnicos).

7. Richardson RJ, Peres JAS, Wanderley JCV, Correia LM, Peres MHM. Pesquisa social: métodos e técnicas. $3^{\mathrm{a}}$ ed. São Paulo: Atlas; 2008.

8. Figueiredo NMA. Método e metodologia na pesquisa científica. $2^{\mathrm{a}}$ ed. São Caetano do Sul (SP): Yendis; 2007.

9. Lakatos EM, Marconi MA. Metodologia do trabalho científico: procedimentos básicos, pesquisa bibliográfica, projeto e relatório, publicações e trabalhos científicos. $7^{\text {a }}$ ed. São Paulo: Atlas; 2009.

10. Gil AC. Como elaborar projetos de pesquisa. $4^{\mathrm{a}}$ ed. São Paulo: Atlas; 2002.

11. Minayo MCS. Pesquisa social: teoria, método e criatividade. $29^{a}$ ed. Petrópolis (RJ): Vozes; 2010.

12. Silva JS, Mariano ZF, Scopel I. A influência do clima urbano na proliferação do mosquito Aedes aegypti em Jataí (GO), na perspectiva da Geografia Médica. HYGEIA Rev Brasileira de Geografia Médica e da Saúde. 2007;2(5):33-49.

13. Verdeal JCR, Costa Filho R, Vanzillotta C, Macedo GL, Bozza FA, Toscano L, et al. Recomendações para o manejo de pacientes com formas graves de dengue. Rev Bras Ter Intensiva. 2011;23(2):125-33.

14. Rio de Janeiro (RJ). Prefeitura. Secretaria Municipal de Saúde e Defesa Civil. Coordenação de Saúde da Família. Protocolos de Enfermagem na atenção primária à saúde. Rio de Janeiro: Conselho Regional de Enfermagem do Rio de Janeiro; 2012.119 p.

15. Silva LAA, Bonacina DM, Andrade A, Oliveira TC. Desafios na construção de um projeto de educação permanente em saúde. Rev Enferm UFSM [Internet]. 2012 [acesso em 2012 out 2] set/dez;2(3):496-506. Disponível em: http://cascavel.ufsm.br/revistas/ojs2.2.2/index.php/reufsm/article/view/5364/pdf

16. Silva VG, Motta MCS, Zeitoune RCG. A prática do enfermeiro na Estratégia Saúde da Família: o caso do município de Vitória/ES. Rev Eletrônica Enferm. 2010 [acesso em 2012 out 2];12(3):441-8. Disponível em: http://www.revenf.bvs.br/pdf/ree/v12n3/05.pdf.

17. Barreto ML, Teixeira MG. Dengue no Brasil: situação epidemiológica e contribuições para uma agenda de pesquisa. Estud Av. 2008;22(64):53-72.

18. Teixeira MG, Costa MCN, Barreto ML. E o dengue continua desafiando e causando perplexidade (editorial). Cad Saúde Pública. 2011;27(5):828-9.

Data de recebimento: $31 / 05 / 2013$

Data de aceite: 04/11/2013

Contato com autor responsável: Maria José Estanislau Daher

Endereço postal: Rua Correa Dutra 158 cob 01, Flamengo, Rio de Janeiro, Brasil, CEP 22250010.

E-mail: maria_daher@ymail.com 